\title{
Multiobjective Nonlinear Symmetric Duality Involving Generalized Pseudoconvexity
}

\author{
Mohamed Abd El-Hady Kassem \\ Mathematical Department, Faculty of Science, Tanta University, Tanta, Egypt \\ E-mail: mohd60371@hotmail.com \\ Received April 8, 2011; revised June 9, 2011; accepted June 16, 2011
}

\begin{abstract}
The purpose of this paper is to introduce second order $(K, F)$-pseudoconvex and second order strongly $(K, F)$ pseudoconvex functions which are a generalization of cone-pseudoconvex and strongly cone-pseudoconvex functions. A pair of second order symmetric dual multiobjective nonlinear programs is formulated by using the considered functions. Furthermore, the weak, strong and converse duality theorems for this pair are established. Finally, a self duality theorem is given.
\end{abstract}

Keywords: Multiobjective Programming, Second-Order Symmetric Dual Models, Duality Theorems, Pseudoconvex Functions, Cones

\section{Introduction}

Duality is an important concept in the study of nonlinear programming. Symmetric duality in nonlinear programming in which the dual of the dual is the primal was first introduced by Dorn [1]. Subsequently Dantzig et al. [2] established symmetric duality results for convex/concave functions with nonnegative orthant as the cone. The symmetric duality result was generalized by Bazaraa and Goode [3] to arbitrary cones. Kim et al. [4] formulated a pair of multiobjective symmetric dual programs for pseudoinvex functions and arbitrary cones. The weak, strong, converse and self duality theorems were established for that pair of dual models.

The study of second order duality is significant due to the computational advantage over first order duality as it provides tighter bounds for the value of the objective function when approximations are used (see Hou and Yang [5], Yang et al. [6,7], Yang et al. [8]).

Hou and Yang [5] introduced a pair of second order symmetric dual non-differentiable programs and second order $F$-pseudoconvex and proved the weak and strong duality theorems for these second order symmetric dual programs under the $F$-pseudoconvex assumption. Suneja et al. [9] formulated a pair of multiobjective symmetric dual programs over arbitrary cones for cone-convex functions. The weak, strong, converse and self-duality theo- rems were proved for these programs. Yang et al. [6] formulated a pair of Wolf type non-differentiable second order symmetric primal and dual problems in mathematical programming. The weak and strong duality theorems were established under second order F-convexity assumptions. Symmetric minimax mixed integer primal and dual problems were also investigated. Khurana [10] introduced cone-pseudoinvex and strongly cone-pseudoinvex functions, and formulated a pair of Mond-Weir type symmetric dual multiobjective programs over arbitrary cones. The duality theorems and the self-dual theorem were established under these functions. Yang et al. [8] proved the weak, strong and converse duality theorems under $F$-convexity conditions for a pair of second order symmetric dual programs. Yang et al. [7] established various duality results for nonlinear programming with cone constraints and its four dual models introduced by Chandra and Abha [11].

In this paper, we present new definitions dealing with second order $(K, F)$-pseudoconvex and second order strongly $(K, F)$-pseudoconvex functions which are a generalized of cone-pseudoconvex and strongly cone-pseudoconvex functions. We suggest a pair of multiobjective nonlinear second order symmetric dual programs. Moreover, we establish the duality theorems using the above generalization of cone-pseudoconvex functions. Finally, a self-duality theorem is given by assuming the skew- 
symmetric of the functions.

\section{Notations and Definitions}

The following conventions for vectors in $R^{n}$ will be used: $x<y \Leftrightarrow x_{i}<y_{i}, i=1,2, \cdots, n$, $x \leqq y \Leftrightarrow x_{i} \leqq y_{i}, i=1,2, \cdots, n$, $x \leq y \Leftrightarrow x_{i} \leqq y_{i}, i=1,2, \cdots, n$ but $x \neq y$.

A general multiobjective nonlinear programming problem can be expressed in the form:

$$
\text { (P): } \min f(x)=\left(f_{1}(x), f_{2}(x), \cdots, f_{m}(x)\right)
$$

subject to $x \in X=\left\{x \in R^{n} \mid g_{j}(x) \leqq 0, j=1,2, \cdots, k\right\}$

where $f: R^{n} \rightarrow R^{m}$ and $g: R^{n} \rightarrow R^{k}$.

Definition 1. A point $\bar{X} \in X$ is said to be an efficient (or a Pareto optimal) solution of problem (P) if there exists no other $x \in X$ such that $f(x) \leq f(\bar{x})$, $\left(f_{i}(x) \leqq f_{i}(\bar{x}), \quad i=1,2, \cdots, m\right.$ but $\left.f(x) \neq f(\bar{x})\right)$.

Recall the following three definitions aiming to give the desired definition (i.e., Definition 5).

Definition 2. [5,7,8] A functional

$F: X \times X \times R^{n} \rightarrow R\left(X \subseteq R^{n}\right)$ is sublinear in its third component if, for all $(x, u) \in X \times X$, and

1) $F\left(x, u ; a_{1}+a_{2}\right) \leqq F\left(x, u ; a_{1}\right)+F\left(x, u ; a_{2}\right) \forall a_{1}, a_{2} \in R^{n}$;

2) $F(x, u ; \alpha a)=\alpha F(x, u ; a) \forall a \in R^{n}, \forall \alpha \in R, \alpha \geqq 0$.

For notational convenience, we write

$$
F_{x, u}(a)=F(x, u ; a) \text {. }
$$

Let $K$ be a closed convex pointed cone in $R^{m}$ with int $K \neq \phi$ and $f: R^{n} \rightarrow R^{m}$ be a differentiable function.

Definition 3. $[4,10,12]$ The polar cone $K^{*}$ of $K$ is defined as

$$
K^{*}=\left\{z \in R^{m} \mid x^{T} z \geqq 0 \forall x \in K\right\} .
$$

Definition 4. [5] The function $f$ is said to be secondorder $F$-pseudoconvex at $u \in X$ if $(x, p) \in X \times R^{n}$,

$$
\begin{aligned}
& F_{x, u}\left[\nabla_{u} f(u)+\nabla_{u u} f(u) p\right] \geqq 0 \\
& \Rightarrow f(x) \geqq f(u)-\frac{1}{2} p^{T} \nabla_{u u} f(u) p .
\end{aligned}
$$

$f$ is second-order $F$-pseudoconcave if $-f$ is second-order $F$-pseudoconvex.

Now, we are in position to give our definitions of second-order $(K, F)$-pseudoconvex functions and secondorder strongly $(K, F)$-pseudoconvex functions.

Definition 5. The function $f$ is said to be second-order $(K, F)$-pseudoconvex at $u \in X$ if $(x, p) \in X \times R^{n}$,

$$
\begin{aligned}
& -F_{x, u}\left[\nabla_{u} f(u)+\nabla_{u u} f(u) p\right] \notin \operatorname{int} K \\
& \Rightarrow-\left[f(x)-f(u)+\frac{1}{2} p^{T} \nabla_{u u} f(u) p\right] \notin \operatorname{int} K ;
\end{aligned}
$$

and the function $f$ is said to be second-order strongly $(K, F)$-pseudoconvex at $u \in X$ if $(x, p) \in X \times R^{n}$,

$$
\begin{aligned}
& -F_{x, u}\left[\nabla_{u} f(u)+\nabla_{u u} f(u) p\right] \notin \operatorname{int} K \\
& \Rightarrow f(x)-f(u)+\frac{1}{2} p^{T} \nabla_{u u} f(u) p \in K .
\end{aligned}
$$

$f$ is second-order $(K, F)$-pseudoconcave if $-f$ is secondorder $(K, F)$-pseudoconvex and $f$ is second-order strongly $(K, F)$-pseudoconcave if $-f$ is second-order strongly $(K, F)$-pseudoconvex.

Remark 1. If $p=0$ and $F_{x, u}(\nabla f(u))=\eta(x, u) \nabla f(u)$ where $\eta: X \times X \rightarrow R^{n},\left(X \subseteq R^{n}\right)$, the second-order strongly $(K, F)$-pseudoconvex functions and second-order $(K, F)$-pseudoconvex functions reduce to strongly $K$ pseudoinvex functions and $K$-pseudoinvex functions defined by Khurana [10].

Remark 2. Every second-order strongly $(K, F)$-pseudoconvex function is second-order $(K, F)$-pseudoconvex but converse is not necessarily true as can be seen from the following example.

Example 1. Let

$$
\begin{aligned}
& K=\left\{(x, y) \mid-4 x \leqq y \leqq-\frac{x}{2}, x>0\right\}, \\
& f(x)=\left(-x^{2}+x, e^{-x}\right), p=1
\end{aligned}
$$

and

$$
F_{x, u}(A)=A\left(x^{3}+u\right)
$$

It can be seen that $f(x)$ is second-order $(K, F)$-pseudoconvex at $u=0$ but $f(x)$ is not second-order strongly $(K, F)$-pseudoconvex at $u=0$ because for $x=1$

$$
-F_{x, u}\left[\nabla_{u} f(u)+\nabla_{u u} f(u) p\right] \notin \operatorname{int} K
$$

and

$$
f(x)-f(u)+\frac{1}{2} p^{T} \nabla_{u и} f(u) p \notin K .
$$

The following example show that a function which is second-order strongly $(K, F)$-pseudoconvex but not second-order $F$-pseudoconvex where $K$ is a closed convex cone.

Example 2. Let

$$
\begin{aligned}
& K=\{(x, y) \mid y \geqq x, y \geqq-x, x \geqq 0\}, \\
& f(x)=\left(-x^{2}+3 x, x^{2}\right), p=1
\end{aligned}
$$

and

$$
F_{x, u}(A)=A\left(x^{3}+u\right) .
$$


Then $f(x)$ is second-order strongly $(K, F)$-pseudoconvex at $u=0$. However, $f(x)$ is not $F$-pseudoconvex at $u=0$, because for $x=3$,

$$
F_{x, u}\left[\nabla_{u} f(u)+\nabla_{u u} f(u) p\right]>0
$$

but

$$
f_{1}(x)>f(u)-\frac{1}{2} p^{T} \nabla_{u u} f(u) p .
$$

We formulate the following multiobjective nonlinear symmetric dual problems:

$$
\text { (SP): } \min f(x, y)-\frac{1}{2} p^{T} \nabla_{y y}\left(\lambda^{T} f\right)(x, y) p
$$

subject to $-\left[\nabla_{y}\left(\lambda^{T} f\right)(x, y)+\nabla_{y y}\left(\lambda^{T} f\right)(x, y) p\right] \in C_{2}^{*}$,

$$
\begin{gathered}
y^{T}\left[\nabla_{y}\left(\lambda^{T} f\right)(x, y)+\nabla_{y y}\left(\lambda^{T} f\right)(x, y) p\right] \geqq 0, \\
\lambda \in K^{*}, x \in C_{1} .
\end{gathered}
$$

(SD): $\max f(u, v)-\frac{1}{2} r^{T} \nabla_{u u}\left(\lambda^{T} f\right)(u, v) r$

subject to $\nabla_{u}\left(\lambda^{T} f\right)(u, v)+\nabla_{u u}\left(\lambda^{T} f\right)(u, v) r \in C_{1}^{*}$,

$$
\begin{gathered}
u^{T}\left[\nabla_{u}\left(\left(\lambda^{T} f\right)(u, v)+\nabla_{u u}\left(\lambda^{T} f\right)(u, v) r\right] \leqq 0,\right. \\
\lambda \in K^{*}, v \in C_{2},
\end{gathered}
$$

where $f: R^{n} \times R^{l} \rightarrow R^{m}$ is a thrice differentiable function of $x$ and $y . C_{1}$ and $C_{2}$ are closed convex cones- with nonempty interiors in $R^{n}$ and $R^{l}$ respectively. For example, the nonnegative orthant $\left\{x \in R^{n} \mid x \geqq 0\right\}$ is a convex cone). $C_{1}^{*}$ and $C_{2}^{*}$ are positive polar cones of $C_{1}$ and $C_{2}$, respectively. $K$ is a closed convex pointed cone in $R^{m}$ such that int $K \neq \phi$ and $K^{*}$ is its positive polar cone. $\nabla_{y}\left(\lambda^{T} f\right)(x, y)$ and $\nabla_{y y}\left(\lambda^{T} f\right)(x, y)$ are the gradient and the Hessian matrix of $\left(\lambda^{T} f\right)(x, y)$ with respect to $y$, respectively.

Similarly, $\nabla_{u}\left(\lambda^{T} f\right)(x, y)$ and $\nabla_{u u}\left(\lambda^{T} f\right)(x, y)$ are the gradient and the Hessian matrix of $\left(\lambda^{T} f\right)(x, y)$ with respect to $u$, respectively.

Observe that if $p=r=0$, then (SP) and (SD) becomes $(\mathrm{P})$ and $(\mathrm{D})$ given by Khurana [8], respectively.

\section{Symmetric Duality}

Now, we establish the symmetric duality theorems for the problems (SP) and (SD) as follows.

Theorem 1. (Weak duality). Let $(x, y, \lambda, p)$ be feasible solution for the problem (SP) and $(u, v, \lambda, r)$ be feasible solution for the problem (SD). Suppose there exist sublinear functionals $F: X \times X \times R^{n} \rightarrow R \quad\left(X \subseteq R^{n}\right)$ and
$G: Y \times Y \times R^{l} \rightarrow R \quad\left(Y \subseteq R^{l}\right)$ satisfying:

$$
\begin{gathered}
F_{x, u}(a)+a^{T} u \in C_{1} \forall a \in C_{1}, \\
G_{v, y}(b)+b^{T} y \in C_{2} \forall b \in C_{2} .
\end{gathered}
$$

Furthermore, assume that either

1) $f(., v)$ is second-order $(K, F)$-pseudoconvex at $u$ and $f(., v)$ is second-order $(K, G)$-pseudoconcave at $y$; or

2) $f(., v)$ is second-order strongly $(K, F)$-pseudoconvex at $u$ and $f(., v)$ is second-order strongly $(K, G)$ pseudoconcave at $y$.

Then

$$
\begin{aligned}
& f(u, v)-\frac{1}{2} r^{T} \nabla_{u u}\left(\lambda^{T} f\right)(u, v) r \\
& -f(x, y)+\frac{1}{2} p^{T} \nabla_{y y}\left(\lambda^{T} f\right)(x, y) p \notin \operatorname{int} K
\end{aligned}
$$

Proof: Suppose the contrary, i.e.,

$$
\begin{aligned}
& f(u, v)-\frac{1}{2} r^{T} \nabla_{u и}\left(\lambda^{T} f\right)(u, v) r \\
& -f(x, y)+\frac{1}{2} p^{T} \nabla_{y y}\left(\lambda^{T} f\right)(x, y) p \in \operatorname{int} K
\end{aligned}
$$

Since $(x, y, \lambda, p)$ is a feasible solution for the problem (SP) and $(u, v, \lambda, r)$ is a feasible solution for the problem (SD), we have:

By the dual constraint (3), the vector

$a=\left[\nabla_{u}\left(\lambda^{T} f\right)(o, v)+\nabla_{u u}\left(\lambda^{T} f\right)(u, v) r\right]$ belongs to $C_{1}^{*}$, and so by (5) we get from (4)

$$
\begin{aligned}
& F_{x, u}\left[\nabla_{u}\left(\lambda^{T} f\right)(u, v)+\nabla_{u u}\left(\lambda^{T} f\right)(u, v) r\right] \\
& \geqq-u^{T}\left[\nabla_{u}\left(\lambda^{T} f\right)(u, v)+\nabla_{u u}\left(\lambda^{T} f\right)(u, v) r\right] \geqq 0 .
\end{aligned}
$$

This gives

$$
-F_{x, u}\left[\nabla_{u}\left(\lambda^{T} f\right)(u, v)+\nabla_{u u}\left(\lambda^{T} f\right)(u, v) r\right] \notin \operatorname{int} K \text {. }
$$

In a similar fashion,

$$
G_{v, y}\left[-\nabla_{y}\left(\lambda^{T} f\right)(x, y)+\nabla_{y y}\left(\lambda^{T} f\right)(x, y) p\right] \geqq 0
$$

for the vector

$$
b=-\left[\nabla_{y}\left(\lambda^{T} f\right)(x, y)+\nabla_{y y}\left(\lambda^{T} f\right)(x, y) p\right]
$$

in $C_{2}^{*}$, and so

$$
-G_{v, y}\left[\nabla_{y}\left(\lambda^{T} f\right)(x, y)+\nabla_{y y}\left(\lambda^{T} f\right)(x, y) p\right] \notin \operatorname{int} K \text {. }
$$

(1) Since the function $f(., v)$ is second-order $(K, F)$ pseudoconvex at $u$, relation (*) implies to

$$
-\left[f(x, v)-f(u, v)+\frac{1}{2} r^{T} \nabla_{u u}\left(\lambda^{T} f\right)(u, v) r\right] \notin \operatorname{int} K .
$$

Similarly from (1) and (6), where 


$$
b=-\left[\nabla_{y}\left(\lambda^{T} f\right)(x, y)+\nabla_{y y}\left(\lambda^{T} f\right)(x, y) p\right] \in C_{2}^{*},
$$

we get

$$
-G_{v, y}\left\{-\left[\nabla_{y}\left(\lambda^{T} f\right)(x, y)+\nabla_{y y}\left(\lambda^{T} f\right)(x, y) p\right]\right\} \notin \operatorname{int} K \text {. }
$$

Also, since the function $f(x,$.$) is second-order (K, G)$ pseudoconcave at $y$ (i.e., $-f(x,$.$) is second-order (K, G)$ pseudoconvex at $y$ ), we have

$$
\left.f(x, v)-f(x, y)+\frac{1}{2} p^{T} \nabla_{y y}\left(\lambda^{T} f\right)(x, y) p\right] \notin \operatorname{int} K
$$

Adding (8) and (9), we get

$$
\begin{aligned}
& f(u, v)-\frac{1}{2} r^{T} \nabla_{u u}\left(\lambda^{T} f\right)(u, v) r \\
& -f(x, y))+\frac{1}{2} p^{T} \nabla_{y y}\left(\lambda^{T} f\right)(x, y) p \notin \operatorname{int} K,
\end{aligned}
$$

this contradicts (7). Hence, the result follows for (1).

(2) From $(*)$ and since the function $f(., v)$ is second-order strongly $(K, F)$-pseudoconvex at $u$, we get

$$
\left[f(x, v)-f(u, v)+\frac{1}{2} r^{T} \nabla_{u и}\left(\lambda^{T} f\right)(u, v) r\right] \notin K .
$$

Also, from (**) and since the function $f(x,$.$) is sec-$ ond-order strongly $(K, G)$-pseudoconcave at $y$ (i.e., $-f(x,$.$) is second-order strongly (K, G)$-pseudoconvex at $y$ ), we get

$$
\left.f(x, y)-f(x, v)+\frac{1}{2} p^{T} \nabla_{y y}\left(\lambda^{T} f\right)(x, y) p\right] \notin K .
$$

Adding (10) and (11), we get

$$
\begin{aligned}
& -f(u, v)+\frac{1}{2} r^{T} \nabla_{u u}\left(\lambda^{T} f\right)(u, v) r \\
& +f(x, y)-\frac{1}{2} p^{T} \nabla_{y y}\left(\lambda^{T} f\right)(x, y) p \notin K,
\end{aligned}
$$

this contradicts (7). Hence, the result follows for (2).

Therefore, the proof is completed.

For the closed convex cones $K$ and $Q$ with nonempty interiors in $R^{m}$ and $R^{k}$, respectively, we will make use the following proposition which gives generalized form of Fritz-John optimality conditions established by suneja et al. [9] for a point to be a weak minimum point of the following multiobjective nonlinear programming problem:

(MONLP): $K-\min f(x)=\left(f_{1}(x), f_{2}(x), \cdots, f_{m}(x)\right)$

$$
\begin{aligned}
& \text { subject to } x \in X=\left\{x \in R^{n} \mid-G(x)\right. \\
& \left.=-\left(g_{1}(x), g_{2}(x), \ldots, g_{k}(x)\right) \in Q\right\}
\end{aligned}
$$

Definition 6. [6,8] A point $\bar{x} \in X$ is said to be a weak minimum point of (MONLP) if for every $x \in X$, $f(\bar{x})-f(x) \notin$ int $K$.

Proposition. [9]. If $\bar{x} \in X$ is a weak minimum point of (MONLP), then there exist $\alpha^{*} \in K^{*}, \beta^{*} \in Q^{*}$ not both zero such that

$$
\begin{gathered}
{\left[\alpha^{*} \nabla f(\bar{x})^{T}+\beta^{*} \nabla G(\bar{x})^{T}\right](x-\bar{x}) \geqq 0 \forall x \in C,} \\
\beta^{* T} G(\bar{x})=0 .
\end{gathered}
$$

Theorem 2. (Strong duality). Let $(\bar{x}, \bar{y}, \bar{\lambda}, \bar{p})$ be a weak minimum point for the problem (SP): fix $\lambda=\bar{\lambda}$ and $r=\bar{r}$ in the problem (SD). Assume that

1) the matrix $\nabla_{y y}\left(\bar{\lambda}^{T} f\right)(\bar{x}, \bar{y})$ is nonsingular,

2) the set $\left\{\nabla_{y} f_{i}(\bar{x}, \bar{y}), i=1,2, \cdots, m\right\}$ is linearly independent,

3) $\left[\nabla_{y}\left(\bar{\lambda}^{T} f\right)(\bar{x}, \bar{y})+\nabla_{y y}\left(\bar{\lambda}^{T} f\right)(\bar{x}, \bar{y}) \bar{p}\right] \neq 0$,

then $(\bar{x}, \bar{y}, \bar{\lambda}, \bar{p}=\bar{r}=0)$ is feasible solution for the problem (SD) and the objective values of the problems (SP) and (SD) are equal.

Furthermore, under the assumptions of Theorem 1, $(\bar{x}, \bar{y}, \bar{\lambda}, \bar{p}=\bar{r}=0)$ is a weak maximum point of the problem (SD).

Proof: Since $(\bar{x}, \bar{y}, \bar{\lambda}, \bar{p})$ is a weak minimum point for the problem (SP), by the Fritz-John conditions of the above proposition, there exist $\alpha \in K^{*}, \beta \in\left(C_{2}^{*}\right)^{*}=C_{2}$, $\gamma \geq 0,(\alpha, \beta, \gamma) \neq 0$, such that for each $x \in C_{1}, \lambda \in K^{*}$, $p \geqq 0$,

$$
\begin{aligned}
& {\left[\alpha^{T} \nabla_{x} f(\bar{x}, \bar{y})+(\beta-\gamma \bar{y})^{T} \nabla_{x y}\left(\bar{\lambda}^{T} f\right)(\bar{x}, \bar{y})+\left(\beta-\gamma \bar{y}-\frac{1}{2} \alpha \bar{p}\right)^{T} \nabla_{x}\left\{\nabla_{y y}\left(\bar{\lambda}^{T} f\right)(\bar{x}, \bar{y}) \bar{p}\right\}\right](x-\bar{x})} \\
& \left.+\left[(\alpha-\gamma \bar{\lambda})^{T} \nabla_{y} f(\bar{x}, \bar{y})+(\beta-\gamma \bar{y}-\gamma \bar{p})^{T} \nabla_{y y}\left(\bar{\lambda}^{T} f\right)(\bar{x}, \bar{y})\right)+\left(\beta-\gamma \bar{y}-\frac{1}{2} \alpha \bar{p}\right)^{T} \nabla_{y}\left\{\nabla_{y y}\left(\bar{\lambda}^{T} f\right)(\bar{x}, \bar{y}) \bar{p}\right\}\right](y-\bar{y}) \\
& +\left[(\beta-\gamma \bar{y})^{T} \nabla_{y} f(\bar{x}, \bar{y})+\left(\beta-\gamma \bar{y}-\frac{1}{2} \alpha \bar{p}\right)^{T} \nabla_{y y} f(\bar{x}, \bar{y}) \bar{p}\right](\lambda-\bar{\lambda}) \\
& +\left[(\beta-\gamma \bar{y}-\alpha \bar{p})^{T} \nabla_{y y}\left(\bar{\lambda}^{T} f\right)(\bar{x}, \bar{y})\right](p-\bar{p}) \geqq 0 \\
& \beta^{T}\left[\nabla_{y}\left(\bar{\lambda}^{T} f\right)(\bar{x}, \bar{y})+\nabla_{y y}\left(\bar{\lambda}^{T} f\right)(\bar{x}, \bar{y}) \bar{p}\right]=0
\end{aligned}
$$




$$
\gamma \bar{y}^{T}\left[\nabla_{y}\left(\bar{\lambda}^{T} f\right)(\bar{x}, \bar{y})+\nabla_{y y}\left(\bar{\lambda}^{T} f\right)(\bar{x}, \bar{y}) \bar{p}\right]=0
$$

Substituting $x=\bar{x} \in C_{1}, \quad y=\bar{y} \in C_{2}$ and $p=\bar{p}$ in the inequality (12), we have

$$
\begin{gathered}
{\left[(\beta-\gamma \bar{y})^{T} \nabla_{y} f(\bar{x}, \bar{y})+\left(\beta-\gamma \bar{y}-\frac{1}{2} \alpha \bar{p}\right)^{T} \nabla_{y y} f(\bar{x}, \bar{y}) \bar{p}\right](\lambda-\bar{\lambda}) \geqq 0 \forall \lambda \in K^{*}} \\
\Rightarrow\left[(\beta-\gamma \bar{y})^{T} \nabla_{y} f(\bar{x}, \bar{y})+\left(\beta-\gamma \bar{y}-\frac{1}{2} \alpha \bar{p}\right)^{T} \nabla_{y y} f(\bar{x}, \bar{y}) \bar{p}\right]=0,
\end{gathered}
$$

this can be written in the following form

$$
(\beta-\gamma \bar{y})^{T}\left[\nabla_{y}\left(\bar{\lambda}^{T} f\right)(\bar{x}, \bar{y})+\nabla_{y y}\left(\bar{\lambda}^{T} f\right)(\bar{x}, \bar{y}) \bar{p}\right]-\frac{1}{2} \alpha \bar{p}^{T} \nabla_{y y}\left(\bar{\lambda}^{T} f\right)(\bar{x}, \bar{y}) \bar{p}=0 .
$$

Subtract (14) from (13), we have

$$
(\beta-\gamma \bar{y})^{T}\left[\nabla_{y}\left(\bar{\lambda}^{T} f\right)(\bar{x}, \bar{y})+\nabla_{y y}\left(\bar{\lambda}^{T} f\right)(\bar{x}, \bar{y}) \bar{p}\right]=0,
$$

then Equation (15) becomes

$$
\alpha \bar{p}^{T} \nabla_{y y}\left(\bar{\lambda}^{T} f\right)(\bar{x}, \bar{y}) \bar{p}=0
$$

Similarly, if $x=\bar{x}, y=\bar{y}$, and $\lambda=\bar{\lambda}$ in Equation (12), we get

$$
\begin{gathered}
(\beta-\gamma \bar{y}-\alpha \bar{p})^{T} \nabla_{y y}\left(\bar{\lambda}^{T} f\right)(\bar{x}, \bar{y})=0 \\
\left\{\gamma^{T}\left[\nabla_{y}\left(\bar{\lambda}^{T} f\right)(\bar{x}, \bar{y})+\nabla_{y y}\left(\bar{\lambda}^{T} f\right)(\bar{x}, \bar{y}) \bar{P}\right]\right\}(y-\bar{y}) \leqq 0 \forall y \in R^{p} \\
\Rightarrow \gamma^{T}\left[\nabla_{y}\left(\bar{\lambda}^{T} f\right)(\bar{x}, \bar{y})+\nabla_{y y}\left(\bar{\lambda}^{T} f\right)(\bar{x}, \bar{y}) \bar{P}\right]=0,
\end{gathered}
$$

using condition (1), we get

$$
\beta=\gamma \bar{y}+\alpha \bar{p}
$$

We claim that $\alpha \neq 0$. Indeed, if $\alpha=0$, then (17) implies

$$
\beta=\gamma \bar{y} .
$$

and from the condition (3), we have

$$
\gamma=0
$$

Therefore, (18) becomes

$$
\beta=0
$$

Hence, $(\alpha, \beta, \gamma)=0$, which contradicts the assumption $(\alpha, \beta, \gamma) \neq 0$. Therefore, $\alpha \neq 0$ and Equation (16) take the form $\bar{p}^{T} \nabla_{y y}\left(\bar{\lambda}^{T} f\right)(\bar{x}, \bar{y}) \bar{p}=0$ and since $\nabla_{y y}\left(\bar{\lambda}^{T} f\right)(\bar{x}, \bar{y})$ is nonsingular (condition (1)) we get

$$
\bar{p}=0
$$

So, Equation (17) becomes

$$
\beta=\gamma \bar{y}
$$

Substituting from Equations (21), (22) and $x=\bar{x}$ in the inequality (12), we get

$$
\begin{gathered}
{\left[(\alpha-\gamma \bar{\lambda})^{T} \nabla_{y} f(\bar{x}, \bar{y})\right](y-\bar{y}) \geqq 0 \forall y \in R^{p}} \\
\Rightarrow(\alpha-\gamma \bar{\lambda})^{T} \nabla_{y} f(\bar{x}, \bar{y})=0 .
\end{gathered}
$$

And since $\nabla_{y} f(\bar{x}, \bar{y})$ is linearly independent (condition (2)), we get

$$
\alpha=\gamma \bar{\lambda}
$$

Using (21), (22) and (23) in (12), we have

$$
\begin{aligned}
& \alpha^{T} \nabla_{x} f(\bar{x}, \bar{y})(x-\bar{x}) \geqq 0,\left(\alpha \in K^{*}\right) \\
& \Rightarrow \nabla_{x} f(\bar{x}, \bar{y})(x-\bar{x}) \geqq 0 \forall x \in C_{1}
\end{aligned}
$$

As $C_{1}$ is closed convex cone, $\bar{x}+x \in C_{1} \forall x \in C_{1}$, hence from (24) and $\lambda \in K^{*}$, we get $x^{T} \nabla_{x}\left(\bar{\lambda}^{T} f\right)(\bar{x}, \bar{y}) \geqq 0 \forall x \in C_{1}$ and by using (21), we get

$$
x^{T}\left[\nabla_{x}\left(\bar{\lambda}^{T} f\right)(\bar{x}, \bar{y})+\nabla_{x x}\left(\bar{\lambda}^{T} f\right)(\bar{x}, \bar{y}) \bar{p}\right] \geqq 0 \forall x \in C_{1},
$$

this implies that

$$
\nabla_{x}\left(\bar{\lambda}^{T} f\right)(\bar{x}, \bar{y})+\nabla_{x x}\left(\bar{\lambda}^{T} f\right)(\bar{x}, \bar{y}) \bar{p} \in C_{1}^{*} .
$$

Similarly, by letting $x=0$ in (24) we have

$$
\bar{x}^{T}\left[\nabla_{x}\left(\bar{\lambda}^{T} f\right)(\bar{x}, \bar{y})+\nabla_{x x}\left(\bar{\lambda}^{T} f\right)(\bar{x}, \bar{y}) \bar{p}\right] \leqq 0 .
$$

Thus $(\bar{x}, \bar{y}, \bar{\lambda}, \bar{p}=0)$ is feasible solution for the problem (SD) and the values of the objective function for the problems (SP) and (SD) are same at $(\bar{x}, \bar{y}, \bar{\lambda}, \bar{p}=0)$. 
We will now show that $(\bar{u}, \bar{v}, \bar{\lambda}, \bar{r}=0)$ is a weak maximum point for the problem (SD).

Suppose not, then there exists a feasible solution $(\bar{u}, \bar{v}, \bar{\lambda}, \bar{r}=0)$ such that

$$
\begin{gathered}
f(\bar{u}, \bar{v})-\frac{1}{2} \bar{r}^{T} \nabla_{u u}\left(\bar{\lambda}^{T} f\right)(\bar{u}, \bar{v}) \bar{r} \\
-f(\bar{x}, \bar{y})+\frac{1}{2} \bar{p}^{T} \nabla_{y y}\left(\bar{\lambda}^{T} f\right)(\bar{x}, \bar{y}) \bar{p} \in \operatorname{int} K,
\end{gathered}
$$

which contradicts the weak duality theorem.

Theorem 3. (Converse duality). Let $(\bar{u}, \bar{v}, \bar{\lambda}, \bar{r})$ be a weak maximum point for the problem (SD). Fix $\lambda=\bar{\lambda}$, $p=\bar{p}$ in the problem (SP). Assume that

1) the matrix $\nabla_{u u}\left(\bar{\lambda}^{T} f\right)(\bar{u}, \bar{v})$ is nonsingular,

2) the set $\left\{\nabla_{u} f_{i}(\bar{u}, \bar{v}), i=1,2, \cdots, m\right\}$ is linearly independent,

3) $\left[\nabla_{u}\left(\bar{\lambda}^{T} f\right)(\bar{u}, \bar{v})+\nabla_{u u}\left(\bar{\lambda}^{T} f\right)(\bar{u}, \bar{v}) \bar{r}\right] \neq 0$,

then $(\bar{u}, \bar{v}, \bar{\lambda}, \bar{r}=0)$ is feasible solution for the problem (SP) and the objective values of the problems (SP) and (SD) are equal.

Furthermore, under the assumptions of Theorem 1, $(\bar{x}, \bar{y}, \bar{\lambda}, \bar{p}=0)$ is a weak minimum point for the problem (SP).

The proof follows on the same lines of Theorem 2 .

\section{Self Duality}

A nonlinear programming problem is said to be self-dual if, when the dual is recast in the form of the primal, the new problem so obtained is the same as the primal problem.

Now we establish the self-duality of the problem (SP).

So, we assume that $n=l, f(x, y)=-f(y, x)$ (i.e., $f$ is skew-symmetric) and $C_{1}=C_{2}, \quad p=r$.

The dual problem (SD) may be rewritten as a minization form:

$$
\text { (SD)': } \min -f(u, v)+\frac{1}{2} r^{T} \nabla_{u u}\left(\bar{\lambda}^{T} f\right)(u, v) r
$$

subject to $\nabla_{u}\left(\lambda^{T} f\right)(u, v)+\nabla_{u u}\left(\lambda^{T} f\right)(u, v) r \in C_{1}^{*}$,

$$
\begin{gathered}
u^{T}\left[\left(\lambda^{T} f\right)(u, v)+\nabla_{u u}\left(\lambda^{T} f\right)(u, v) r\right] \leqq 0, \\
\lambda \in K^{*}, v \in C_{2} .
\end{gathered}
$$

Since $f(u, v)=-f(v, u), \quad \nabla_{u} f(u, v)=-\nabla_{v} f(v, u)$ and $\nabla_{u u} f(u, v)=-\nabla_{v v} f(v, u)$, the above dual problem (SD)' reduces to

$$
\text { (SD)": } \min f(v, u)-\frac{1}{2} r^{T} \nabla_{v v}\left(\bar{\lambda}^{T} f\right)(v, u) r
$$

subject to $-\left[\nabla_{v}\left(\lambda^{T} f\right)(v, u)+\nabla_{v v}\left(\lambda^{T} f\right)(v, u) r\right] \in C_{1}^{*}$,

$$
\begin{gathered}
u^{T}\left[\nabla_{v}\left(\lambda^{T} f\right)(v, u)+\nabla_{v v}\left(\lambda^{T} f\right)(v, u) r\right] \geqq 0, \\
\lambda \in K^{*}, v \in C_{2} .
\end{gathered}
$$

Therefore, this dual problem (SD)' is formally identical to the primal problem (SP), that is, the objective and constraint functions of the problems (SP) and (SD)" are identical. Hence, this problem is self dual. Consequently, the feasibility point $(\bar{x}, \bar{y}, \bar{\lambda}, \bar{p}=\bar{r}=0)$ for the primal problem (SP) implies the feasibility point $(\bar{y}, \bar{x}, \bar{\lambda}$, $\bar{p}=\bar{r}=0$ ) for the dual problem (SD) and vice versa.

Theorem 4. (Self duality). Under the assumptions of the weak duality theorem and the point $(\bar{x}, \bar{y}, \bar{\lambda}, \bar{p}=0)$ is a weak minimum point for the problem (SP), we assume that

1) the primal problem (SP) is self dual,

2) the matrix $\nabla_{y y}\left(\bar{\lambda}^{T} f\right)(\bar{x}, \bar{y})$ is nonsingular,

3) the set $\left\{\nabla_{y} f_{i}(\bar{x}, \bar{y}), i=1,2, \cdots, m\right\}$ is linearly independent,

$$
\text { 4) }\left[\nabla_{y}\left(\bar{\lambda}^{T} f\right)(\bar{x}, \bar{y})+\nabla_{y y}\left(\bar{\lambda}^{T} f\right)(\bar{x}, \bar{y}) \bar{p}\right] \neq 0 \text {, }
$$

then $(\bar{x}, \bar{y}, \bar{\lambda}, \bar{p}=0)$ is a weak minimum point and a weak maximum point, respectively for both the problems (SP) and (SD) and the common optimal value is zero.

Proof: From the strong duality theorem $(\bar{x}, \bar{y}, \bar{\lambda}, \bar{p}=0)$ is a weak maximum point for the problem (SD) and the optimal values of the problems (SP) and (SD) are identical. By using the self duality, we have $(\bar{x}, \bar{y}, \bar{\lambda}, \bar{p}=0)$ is feasible for both problems (SP) and (SD) and using the theorems 1-3, we get that it is optimal for both the problems (SP) and (SD).

To show that the common optimal value is zero, since $f$ is skew symmetric, we have

$$
\begin{aligned}
f(\bar{x}, \bar{y}) & =-f(\bar{x}, \bar{y}), \\
\nabla_{y y} f(\bar{x}, \bar{y}) & =-\nabla_{x x} f(\bar{x}, \bar{y}) .
\end{aligned}
$$

Hence,

$$
\begin{aligned}
& f(\bar{x}, \bar{y})-\frac{1}{2} \bar{p}^{T} \nabla_{y y}\left(\bar{\lambda}^{T} f\right)(\bar{x}, \bar{y}) \bar{p} \\
& =f(\bar{y}, \bar{x})-\frac{1}{2} \bar{p}^{T} \nabla_{x x}\left(\bar{\lambda}^{T} f\right)(\bar{y}, \bar{x}) \bar{p} \\
& =-f(\bar{x}, \bar{y})+\frac{1}{2} \bar{p}^{T} \nabla_{y y}\left(\bar{\lambda}^{T} f\right)(\bar{x}, \bar{y}) \bar{p},
\end{aligned}
$$

and so

$$
\begin{aligned}
& f(\bar{x}, \bar{y})-\frac{1}{2} \bar{p}^{T} \nabla_{y y}\left(\bar{\lambda}^{T} f\right)(\bar{x}, \bar{y}) \bar{p} \\
& =f(\bar{y}, \bar{x})-\frac{1}{2} \bar{p}^{T} \nabla_{x x}\left(\bar{\lambda}^{T} f\right)(\bar{y}, \bar{x}) \bar{p}=0 .
\end{aligned}
$$

\section{Conclusions}

A pair of symmetric dual programs has been formulated 
by considering the optimization with respect to an arbitrary cone under the assumptions of second order $(K, F)$ pseudoconvex and second order strongly $(K, F)$-pseudoconvex functions. The results may be further generalized by relaxing the condition of cone-pseudoconvex functions to cone-pseudobonvex functions.

\section{References}

[1] W. S. Dorn, "A Symmetric Dual Theorem for Quadratic Programs," Journal of the Operations Research Society of Japan, Vol. 2, 1960, pp. 93-97.

[2] G. B. Dantzig, E. Eisenberg and R. W. Cottle, "Symmetric Dual Nonlinear Programs," Pacific Journal of Mathematics, Vol. 15, No. 3, 1965, pp. 809-812.

[3] M. S. Bazaraa and J. J. Goode, “On Symmetric Duality in Nonlinear Programming,” Operation Research, Vol. 21, No. 1, 1973, pp. 1-9. doi:10.1287/opre.21.1.1

[4] D. Sang Kim, Y. B. Yun and W. J. Lee, "Multiobjective Symmetric Duality with Cone Constraints," European Journal of Operational Research, Vol. 107, No. 3, 1998, pp. 686-691. doi:10.1016/S0377-2217(97)00322-6

[5] S. H. Hou and X. M. Yang, "On Second-Order Symmetric Duality in Non-Differentiable Programming,” Journal of Mathematical Analysis and Applications, Vol. 255, 2001, pp. 491-498. doi:10.1006/jmaa.2000.7242

[6] X M. Yang, X. Q. Yang and K. L. Teo, "Non-Differentiable Second Order Symmetric Duality in Mathematical
Programming with F-Convexity,” European Journal of Operational Research, Vol. 144, 2003, pp. 554-559. doi:10.1016/S0377-2217(02)00156-X

[7] X. M. Yang, X. Q. Yang and K. L. Teo, "Converse Duality in Nonlinear Programming with Cone Constraints," European Journal of Operational Research, Vol. 170, 2006, pp. 350-354. doi:10.1016/j.ejor.2004.05.028

[8] X. M. Yang, X. Q. Yang, K. L. Teo and S. H. Hou, "Multiobjective Second Order Symmetric with F-Convexity," European Journal of Operational Research, Vol. 165, No. 3, 2005, pp. 585-591. doi:10.1016/j.ejor.2004.01.028

[9] K. Suneja, S. Aggarwal and S. Davar, "Multiobjective Symmetric Duality involving Cones,” European Journal of Operational Research, Vol. 141, No. 3, 2002, pp. 471479. doi:10.1016/S0377-2217(01)00258-2

[10] S. Khurana, "Symmetric Duality in Multiobjective Programming involving Generalized Cone-Invex Functions," European Journal of Operational Research, Vol. 165, No. 3, 2005, pp. 592-597. doi:10.1016/j.ejor.2003.03.004

[11] S. Chandra and A. Abha, "A Note on Pseudo-Invex and Duality in Nonlinear Programming," European Journal of Operational Research, Vol. 122, No. 1, 2000, pp. 161165. doi:10.1016/S0377-2217(99)00076-4

[12] M. Kassem, "Higher-Order Symmetric Duality in Vector Optimization Problem involving Generalized Cone-Invex Functions,” Applied Mathematics and Computation, Vol. 209, No. 2, 2009, pp. 405-409. doi:10.1016/j.amc.2008.12.063 